\title{
The power of experimental models for understanding the genetic basis for superior endurance running
}

\begin{abstract}
The genetic basis for superior endurance running still remains elusive. The usage of experimental animal models have been shown to be of considerable help understanding in more detail the role and function of single genes. Due to the starling gene homology between mice and humans, mouse knockout technology has great advantages and is fundamental in biomedical research. The development and physiology of the mouse is similar with those in humans, being relevant for the proper study of the musculoskeletal system and therefore important for superior endurance running.
\end{abstract}

Keywords: Musculoskeletal system, Muscles, Tendons, Endurance running, Mouse knockout models, Endurance training, Forced exhaustive tests, Tenomodulin
Volume I Issue 2 - 2017

Manuel Delgado Caceres, Denitsa Docheva
Experimental Trauma Surgery, Department of Trauma Surgery,

University Regensburg Medical Centre, Germany

Correspondence: Denitsa Docheva, Experimental Trauma Surgery, Department of Trauma Surgery, University Regensburg Medical Centre, Germany, Tel 0049 94I 943 I605,

Email denitsa.docheva@ukr.de

Received: May 30, 2017 | Published: June 02, 2017
Abbreviations: ER, endurance running; Tnmd, tenomodulin; $\mathrm{KO}$, knockout; ILK, integrin-linked kinase

\section{Introduction}

Many studies related to running have been undertaken over the past decades. The biomechanics behind it have been well investigated and extensively described; however, the basis on which genetics might influence endurance running (ER) still remains elusive. ER is defined as running a non-determined distance over extended time periods using aerobic metabolism and is one of the most complex human features because a considerable amount of body systems have to function finely together (musculoskeletal, respiratory, cardiovascular, etc.). ${ }^{1,2}$ Even though humans are known to be rather average speed runners (compared to other mammals), they stand out in ER. ${ }^{1}$

In this short opinion article, we will focus mainly on experimental animal models related to the study of musculoskeletal system (muscles and tendons) and their relevance regarding superior ER. The main function of muscle is force and motion production and this is achieved by coordinated contractions. Muscles can only work properly if the cardiovascular system delivers enough oxygen. ${ }^{2}$ The main function of tendons is to connect muscles to bone. Tendons function like springs storing and transmitting the muscle-generated force to the bone, enabling movement.

Experimental studies have helped researchers to comprehend in a more accurate manner, how different tissues develop (ontogeny), their composition and their functionality. The most obvious approach to investigate a gene is by generating a knockout (KO) ("loss of gene function") and by validating the outcome via whole animal phenol typing. We will compactly describe, using two examples, how the usage of experimental mouse models supports the improved understanding of ER at a molecular level. The role of integrin-linked kinase (ILK) in skeletal muscles and tenomodulin (TNMD/Tnmd) in tendons was assessed using a conditional $\mathrm{KO} \mathrm{model}^{3}$ and a constitutive KO mouse model, respectively. ${ }^{4}$

Integrin-linked kinase is a protein highly expressed in skeletal muscle, more precisely at myotendinous junctions (MTJs). The principal function of MTJs is to bind myofibers to tendons and therefore it is relevant for proper force transmission. Transgenic mice with a skeletal muscle-restricted deletion of ILK (HSACre-ILK mice) did not show an apparent phenotype at birth, but after 3 weeks, an abnormal walking pattern with a considerably shorter stride length was detected. ${ }^{3}$ In order to test if mechanical stress had an effect on muscle integrity, 5-mo-old HSACre-ILK mice were subjected to daily treadmill exercise $(60 \mathrm{~min}$. at $18 \mathrm{~m} / \mathrm{min}$ with an upward inclination of $10^{\circ}$ ). Initially, mutant and control animals maintained the required speed during the entire training session. After the first three weeks, training had to be stopped because transgenic animals were unable to run without breaks. Ultra structural analysis revealed that training and running induced alterations in myofibers of HAS-Cre-IKL mice, generating muscle damage and subsequently mild progressive muscular dystrophy restricted to the MTJs. ${ }^{3}$

Tnmd is a well-accepted gene marker for mature tendons and ligaments and its precise function has been studied since its discovery in 2001. It has been recently reported that Tnmd is needed for optimal running performance. ${ }^{4}$ Mice lacking Tnmd failed in forced endurance running tests (daily treadmill exercise for $60 \mathrm{~min}$. at $10 \mathrm{~m} / \mathrm{min}$ with a declination of $10^{\circ}$ ). Transgenic animals ran significantly less time, dropped earlier from the treadmill, and were unable to maintain high speed; the running performance further worsened with training. In contrast, training showed beneficial effects on wild type control animals. This evidence strongly suggests that Tnmd is an important gene required for proper tendon tissue adaptation and there for epivotal for superior endurance running. ${ }^{4}$

\section{Conclusion}

The number of published articles related to the study of the genetic basis of running has increased slowly over the last years. Still, further investigation is necessary to accurately explain which genes play a concrete role in endurance running and athletic activities. The usage of cross comparative studies, different training regimes and a variety of mice strains should additionally help to decipher the complex genetic program behind ER; opening doors of opportunity for the translation into the human situation. 


\section{Acknowledgements}

D. D. acknowledges the German Research Foundation (Grant Nr. DO1414/3-1). M. D-C. and D. D. thank Dr. G. Pattappa for reading the article.

\section{Conflict of interest}

Author declares there is no conflict of interest in publishing the article.

\section{References}

1. Bramble DM, Lieberman DE. Endurance running and the evolution of Homo. Nature. 2004;432(7015):345-352.
2. Lerman I, Harrison BC, Freeman K, et al. Genetic variability in forced and voluntary endurance exercise performance in seven inbred mouse strains. J Appl Physiol. 2002;92(6):2245-2255.

3. Wang HV, Chang LW, Brixius K, et al. Integrin-linked kinase stabilizes myotendinous junctions and protects muscle from stress-induced damage. J Cell Bio. 2008;180(5):1037-1049.

4. Dex S, AlbertonP, Willkomm L, et al. Tenomodulin is Required for Tendon Endurance Running and Collagen I Fibril Adaptation to Mechanical Load. EBioMedicine. 2017. p. 1-15. 